\title{
Review of: "Next generation live-attenuated yellow fever vaccine candidate: Safety and immuno-efficacy in small animal models"
}

\author{
Monique Lafon ${ }^{1}$, CHRISTOPHE PREHAUD $^{1}$ \\ 1 Pasteur Institute
}

Potential competing interests: The author(s) declared that no potential competing interests exist.

Yellow Fever (YF) is a lethal mosquito-borne flavivirus disease causing viral hemorrhagic fever characterized by hepatitis, jaundice, renal failure, hemorrhages and shock. The case fatality rate is high (20-50\%) with likely 200000 cases and 30000 death per year. It affects the tropical area in Africa and South America, both endemically and epidemically. Monkeys and mosquitoes are natural reservoirs, and both proved to be difficult to control. By any means, the most effective prevention strategy of the disease reside in the human vaccination. This has been brilliantly achieved for years by the use of live-attenuated vaccine, the YF-17D vaccine produced in chicken embryonated eggs, which by a single dose confers protective long-term immunity. The production in embryonated eggs is a highly controlled efficient process. However, during the last outbreak in Africa and South America, and the resulting increase in the demand for vaccines, it became clear that the supplies of pathogen-free embryonated eggs storage was a bottleneck for rapid scale up of the production (ref UNICEF YFV vaccine current supply outlook 2016). The question arose whether it was time to replace the production of the YF vaccine on embryonated eggs by a production on cell culture [1]?

The development of cell cultures for the production of viral vaccines began in the 1930s with the production of polio vaccine (Salk). It continued with the production in the 1960s by the Wistar Institute of a primary human cell line, the WI-38 diploid human cell line, allowing the development of the first rabies vaccine on cell culture [2]. The next step of improvement was the replacement in the 1980s of the primary cells, by continuous cell lines, such as the Vero cells obtained from the kidney of an African green monkey. The validation of a Vero Master Cell bank allows the first production of polio and rabies vaccines on Vero cells [3]. The confidence in Vero cells, (absence of tumorigenicity, absence of adventitious viruses), and the technical improvements, (adaptation of Vero cells to the culture in fermentor and in serum free medium), have made Vero cells in a few decades, the highly valuable cell culture platform of choice for viral vaccine production. This allowed the development of a large range of vaccines or vaccine candidates targeting different viral virus diseases [4] including a live-attenuated Japanese Encephalitis (JE) virus vaccine based on a chimeric Yellow Fever-Japanese encephalitis virus (ChimeriVax-JE) vaccine. In the article entitled "Next generation live-attenuated yellow fever vaccine candidate : safety and immune-efficacy in small animal models" [5], Fabienne Piras-Douce and colleagues described the 
generation and preclinical profile of a live-attenuated YF-17D vaccine candidate, vYF, adapted for growth in Vero cells in serum free medium.

The safety of immunogenicity of this candidate vaccine in two rodent animal models (mice and hamsters) prompted Sanofi-Pasteur to start in 2021 a clinical trial (NCT04142086) to test safety and immunogenicity of 3 dosing regimens in adult healthy volunteers- (73 participants). In the hypothesis the performance of the VYF candidate is not lesser than those of the YF-VAX, it could pave the way of an improved YF fever vaccine. The benefits of the VYF vaccine produced on Vero cells in serum free medium compared to YFVAX vaccine produced in embryonated eggs are to i) avoid vaccination complications due to hypersensitivity reactions to egg proteins, ii) eliminate substances of animal origin and iii) stabilize the variation between lots.

However, the VYF vaccine candidate remains a live-attenuated vaccine as is the YF-VAX. The risk of developing vaccine-associated diseases, will not be eliminated with the vYF vaccine, because it will have the capacity to multiply in the organs of the vaccines.

The live-attenuated vaccine is well-tolerated and post-vaccination complications are infrequent Nevertheless, severe adverse events can occur after YF vaccination; vaccine Associated Viscerotropic Disease (YEL-AVD) or vaccine Associated Neurotropic Disease (YEL-AND). YEL-AND is characterized by encephalomyelitis or meningoencephalitis [6], which frequency is higher than those of YEL-AVD (0.8 and 0.3 per 100000 doses distributed for YEL-AND and YEL-AVD respectively) and increases with age (2.2 per 100000 vaccines for patient over 60 years old, compared to 0.8 per 100000 vaccines younger than 60). Origin of YEL-AND is not clear [7]. One hypothesis is that they could result from the appearance of variants during the preparation of the vaccine seed lots. RNA viruses have a high mutation rate, Many RNA virus polymerases lack proofreading and correcting activity, which contributes to a high mutation rate during viral replication. The short replication cycle of these viruses rapidly generates a heterogeneous viral population, called viral quasi-species, which contains a multitude of variants that can be associated with different phenotypes. Such variants would have acquired the possibility of entering the brain, propagating into the nervous tissues and causing neurotoxic reactions (neurovirulence) [8]. Such a possibility is supported by the isolation from a fatal case of vaccine associated viral encephalitis of a neuroinvasive/neurotropic and neurovirulent variant which sequence was distinct from those of the vaccine strain (YF-17D-204) [9].

The use of a vaccine not able to replicate in the host, an inactivated vaccine, should circumvent the occurrence of these adverse events, and make the contraindications in infants under 9 months of age, pregnant and nursing women, or immunosuppressed and elderly persons disappear. We can hope that this will be the next step for the authors in their quest for yellow fever vaccine improvement.

References

1. Tomashek KM, Challberg M, Nayak SU, Schiltz HF: Disease Resurgence, Production Capability Issues and Safety Concerns in the Context of an Aging Population: Is There a Need for a New 
Yellow Fever Vaccine? Vaccines (Basel) 2019, 7.

2. Plotkin SA: Rabies vaccine prepared in human cell cultures: progress and perspectives . Rev Infect Dis 1980, 2:433-448.

3. Montagnon BJ: Polio and rabies vaccines produced in continuous cell lines: a reality for Vero cell line. Dev Biol Stand 1989, 70:27-47.

4. Barrett PN, Mundt W, Kistner O, Howard MK: Vero cell platform in vaccine production: moving towards cell culture-based viral vaccines. Expert Rev Vaccines 2009, 8:607-618.

5. Piras-Douce F, Raynal F, Raquin A, Girerd-Chambaz Y, Gautheron S, Sanchez MEN, Vangelisti M, Mantel $\mathrm{N}$ : Next generation live-attenuated yellow fever vaccine candidate: Safety and immuno-efficacy in small animal models. Vaccine 2021, 39:1846-1856.

6. Cottin P, Niedrig M, Domingo C: Safety profile of the yellow fever vaccine Stamaril(R): a 17-year review. Expert Rev Vaccines 2013, 12:1351-1368.

7. Barrett $A D$, Teuwen DE: Yellow fever vaccine - how does it work and why do rare cases of serious adverse events take place? Curr Opin Immunol 2009, 21:308-313.

8. Bakoa F, Prehaud C, Beauclair G, Chazal M, Mantel N, Lafon M, Jouvenet N: Genomic diversity contributes to the neuroinvasiveness of the Yellow fever French neurotropic vaccine. NPJ Vaccines 2021, 6:64.

9. Jennings AD, Gibson CA, Miller BR, Mathews JH, Mitchell CJ, Roehrig JT, Wood DJ, Taffs F, Sil BK, Whitby $\mathrm{SN}$, et al.: Analysis of a yellow fever virus isolated from a fatal case of vaccine-associated human encephalitis. J Infect Dis 1994, 169:512-518. 